\title{
Vertical profile reconstruction with Pol-InSAR data of a subpolar glacier
}

\author{
Jayanti J. Sharma*, Irena Hajnsek*, Konstantinos P. Papathanassiou* \\ * Microwaves and Radar Institute, German Aerospace Center \\ 82230 Wessling, Germany \\ Email: jayanti.sharma@dlr.de, irena.hajnsek@dlr.de, kostas.papathanassiou@dlr.de
}

\begin{abstract}
The last decade has seen an increasing demand for accurate mapping and wide-coverage monitoring of glaciers and ice sheets in order to measure and predict their response to global climate change and their contribution to sea level rise. This in turn requires a more complete understanding of their properties including topography, accumulation rates and vertical profiles. One promising new technique for vertical profile reconstruction using polarimetric interferometric SAR (Pol-InSAR) data is Polarization Coherence Tomography (PCT) and for the first time, PCT is adapted here to a glacier scenario. The inversion algorithm to reconstruct vertical ice profiles is applied to both simulated data to assess its accuracy and sensitivity to input parameters, and to airborne Pol-InSAR data at L- and P-band and InSAR data at $X$-band collected using DLR's E-SAR system over the Austfonna ice cap in Svalbard, Norway.
\end{abstract}

\section{INTRODUCTION}

Over the last few decades the use of satellite remote sensing has greatly advanced the field of glaciology, although methods are still lacking in measuring variations in the vertical profiles of glacier firn. In situ studies can measure the glacier subsurface in great detail, but the data acquisitions are limited both spatially and temporally, and the harsh climate and inaccessibility of the polar regions make field measurements particularly difficult.

The PCT (Polarization Coherence Tomography) radarimaging technique [1] estimates vertical profiles by assuming that the unknown profile can be well-approximated by a low-order Legendre polynomial. This technique employs measurements of the interferometric coherence to estimate the Legendre polynomial coefficients and reconstruct the vertical profile of a volume. Advantages of the PCT approach are that it does not make assumptions regarding relationships between scattering in different polarizations and it has the ability to map profiles which do not strictly follow the exponential extinction assumption required by e.g. the Random volume Over Ground (RVoG) [2] model. PCT can thus be used to verify assumptions in an underlying model [1]. The PCT method has shown promise in reconstructing the vertical profiles of forest [1] and agricultural volumes [3], and in this paper the PCT method is adapted to a glacier scenario.

\section{PCT FOR A GLACIER GEOMETRY}

Here we adapt the PCT equations from [1] for a land-ice scenario. The glacier case differs from the vegetation scenario in that at microwave wavelengths and for our side-looking geometry glaciers can be considered to be infinitely thick [4], surface scattering (if significant) occurs at the top instead of at the bottom of the volume, and refraction effects must be considered.

\section{A. Input parameters for PCT}

PCT is based on a Legendre series expansion of the unknown profile over a finite interval [1] requiring estimates of the ground topography and volume extent. In the landice case the surface topography is the upper bound of the scattering volume. We apply the PCT method only to the areas immediately surrounding reflectors deployed on the ice's surface, using the reflector locations as the required surface reference points.

To estimate the effective volume depth seen by the radar, we apply the two-layer scattering model described in [5] consisting of a ground layer overlying an ice volume. We model the ice volume as a semi-infinite half-space extending from the surface (located at $z=0$ ) downwards and consisting of a uniform distribution of scatterers with dielectric constant $\sqrt{\varepsilon} \approx 2.8$ [5]. As derived in [5], the complex coherence after compensation for range spectral decorrelation is given by:

$$
\gamma=e^{j \phi_{0}} \frac{\left(1+\frac{j d_{\mathrm{pen}} k_{z \mathrm{vol}}}{2}\right)^{-1}+m}{1+m},
$$

where $j$ is the imaginary number, $\phi_{0}$ the topographic phase, $m$ the ground-to-volume scattering ratio, and $d_{\text {pen }}$ the penetration depth into the ice volume at which the one-way backscattered power falls to $1 / e$ or $\approx 38 \%$ [6]. The vertical wave number in the volume $k_{z \mathrm{vol}}$ is defined as:

$$
k_{z \mathrm{vol}}=\frac{4 \pi \sqrt{\varepsilon}}{\lambda} \frac{\Delta \theta_{r}}{\sin \theta_{r}},
$$

where $\lambda$ is the wavelength in free space, $\theta_{r}$ is the refracted incidence angle in the ice volume, and $\Delta \theta_{r}$ is the difference in look angles from each antenna in the volume. Using Eq. 1 a look-up table of $\gamma$ as a function of $m$ and $d_{\text {pen }}$ may be constructed and compared against observed coherence values for an estimate of $d_{\text {pen }}$. We approximate the depth seen by the radar $d_{\mathrm{vol}}$ as the penetration depth multiplied by a factor $C$. 


\section{B. Single-baseline PCT for a glacier geometry}

To reconstruct the vertical profile in a glacier volume for a single-baseline scenario we begin with the equation for the complex coherence in an infinite volume (after correction of range spectral decorrelation) given by [5]:

$$
\gamma=e^{j \phi_{0}} \cdot \frac{\int_{-\infty}^{0} f(z) e^{-j k_{z \mathrm{vol}} z} d z}{\int_{-\infty}^{0} f(z) d z}
$$

where $f(z)$ is the unknown vertical structure function representing the variation in radar cross section with depth. To perform a Fourier-Legendre decomposition, we replace the integral bounds $(-\infty, 0]$ with $\left[d_{\mathrm{vol}}, 0\right]$ and normalize the integral limits to $[-1,1]$ using a change of variable such that:

$$
\gamma=e^{j \phi_{0}} e^{-j k_{p}} \cdot \frac{\int_{-1}^{1}\left(1+f\left(z^{\prime}\right)\right) e^{-j k_{p} z^{\prime}} d z^{\prime}}{\int_{-1}^{1}\left(1+f\left(z^{\prime}\right)\right) d z^{\prime}},
$$

with

$$
\begin{aligned}
z^{\prime} & =\frac{-2 z}{d_{\mathrm{vol}}}+1 \\
f\left(z^{\prime}\right) & =f(z)-1 \\
k_{p} & =-k_{z \mathrm{vol}} \frac{d_{\mathrm{vol}}}{2},
\end{aligned}
$$

where $z^{\prime}$ and $f\left(z^{\prime}\right)$ are the normalized depth and normalized structure function, and $k_{p}$ is the scaled depth and $k_{z \mathrm{vol}}$ product. It is assumed that $f\left(z^{\prime}\right)$ can be represented by an $m$ th order Legendre series such that:

$$
f\left(z^{\prime}\right)=\sum_{n=0}^{m} a_{n} P_{n}\left(z^{\prime}\right)
$$

where $P_{n}$ are the Legendre polynomials and the coefficients $a_{0}, a_{1}$, etc. are given by [1]:

$$
a_{n}=\frac{2 n+1}{2} \int_{-1}^{1} f\left(z^{\prime}\right) P_{n}\left(z^{\prime}\right) d z^{\prime} .
$$

The expansion of $f\left(z^{\prime}\right)$ up to a 2 nd order Legendre series and insertion into Eq. 4 follows that of [1] to give a decomposition of coherence into:

$$
\gamma=e^{j \phi_{0}} e^{-j k_{p}} \cdot \frac{\left(1+a_{0}\right) f_{0}+a_{1} f_{1}+a_{2} f_{2}}{\left(1+a_{0}\right)},
$$

where the functions $f_{n}$ are given by:

$$
\begin{aligned}
& f_{0}=\frac{\sin k_{p}}{k_{p}} \quad f_{1}=j\left(\frac{\sin k_{p}}{k_{p}^{2}}-\frac{\cos k_{p}}{k_{p}}\right) \\
& f_{2}=\frac{3 \cos k_{p}}{k_{p}^{2}}-\left(\frac{6-3 k_{p}^{2}}{2 k_{p}^{3}}+\frac{1}{2 k_{p}}\right) \sin k_{p} .
\end{aligned}
$$

Only the relative structure function can be determined because of the arbitrary scaling factor $\left(1+a_{0}\right)$ in Eq. 10. We subsequently normalize the coefficients in Eq. 10 such that $a_{00}=1$ and $a_{n 0}=a_{n} /\left(1+a_{0}\right)$ for $n>0$. Using the fact that $f_{0}$ and $f_{2}$ are purely real and $f_{1}$ is purely imaginary, we define coherence $\gamma_{k}=\gamma e^{-j \phi_{0}} e^{j k_{p}}$ (normalized by topographic phase and $k_{p}$ ) and rewrite Eq. 10 as a linear system of equations in matrix form $[F] \boldsymbol{a}=\boldsymbol{b}[1]$ :

$$
\left[\begin{array}{ccc}
1 & 0 & 0 \\
0 & \operatorname{Im}\left(f_{1}\right) & 0 \\
0 & 0 & f_{2}
\end{array}\right]\left[\begin{array}{l}
a_{00} \\
a_{10} \\
a_{20}
\end{array}\right]=\left[\begin{array}{c}
1 \\
\operatorname{Im}\left(\gamma_{k}\right) \\
\operatorname{Re}\left(\gamma_{k}\right)-f_{0}
\end{array}\right] .
$$

An estimate of the set of unknown real coefficients $a_{n 0}$ is obtained by inverting this relation $\left(\hat{\boldsymbol{a}}=[F]^{-1} \boldsymbol{b}\right)$. The coefficients may then be used to generate an estimate of the unknown vertical structure profile $f\left(z^{\prime}\right)$ from Eq. 8. Because a single baseline provides one complex coherence, two unknown parameters $\left(a_{10}\right.$ and $a_{20}$ ) can be estimated providing a firstorder approximation of the profile.

\section{Simulation of Glacier PCT}

In this section we examine through simulation the ability of Legendre polynomials to reconstruct simple vertical structure functions as well as to determine the sensitivity of the estimated profiles to errors in the input parameters $\phi_{0}$ and $d_{\text {pen. }}$. We consider the set of true profiles built from combinations of exponential and Gaussian functions having the general form [1]:

$$
f(z)=m_{1} e^{\frac{2 \kappa_{e}}{\cos \theta_{r}} z}+m_{2} e^{-g^{2} z^{2}},
$$

where $\kappa_{e}$ is the extinction $\left(\kappa_{e}=-\cos \theta_{r} / d_{\text {pen }}\right)$ under the assumption of a uniform volume [5], and $g$ determines the width of the Gaussian function representing the ground response. Let the integrated ground-to-volume scattering ratio be given by $m$ such that:

$$
m=\frac{\int m_{2} e^{-g^{2} z^{2}} d z}{\int m_{1} e^{\frac{2 \kappa_{e}}{\cos \theta} z} d z} .
$$

\section{A. Ability of Legendre polynomials to reconstruct $f(z)$}

Beginning with the simplest case of a purely exponential profile $\left(m_{1}=1, m_{2}=0\right)$, we derive the Legendre series approximation for a second-order expansion using Eqs. 8 and 9. Results for $\kappa_{e}=0.3 \mathrm{~dB} / \mathrm{m}$ (an inverted extinction at Lband in the experimental data) and $\kappa_{e}=1.0 \mathrm{~dB} / \mathrm{m}$ (an inverted extinction at X-band) at incidence angle $\theta=40^{\circ}$ are shown in Fig. 1. For both curves the second-order Legendre expansion (whose coefficients can be estimated with a single InSAR baseline) follows the true profile quite closely. The effect of an added ground component with ground-to-volume ratio $m=-$ $3 \mathrm{~dB}$ and Gaussian width parameter $g=\left|0.01 d_{\mathrm{vol}}\right|$ is shown in Fig. 2. The second-order Legendre expansion is no longer as good a fit at the lower extinction although the general trend is still correct. A higher-order Legendre polynomial expansion requiring additional InSAR baselines would be required to obtain an estimated structure profile closer to the true $f(z)$.

\section{B. Performance analysis}

Next we consider the influence of stochastic errors in the input parameters on the estimated vertical profiles. An error in penetration depth will alter the rescaling of the integrals in Eqs. 4 to 7, where the influence of an error of $\pm 10 \%$ in $d_{\text {pen }}$ for the volume and volume-plus-ground scenarios is plotted in 


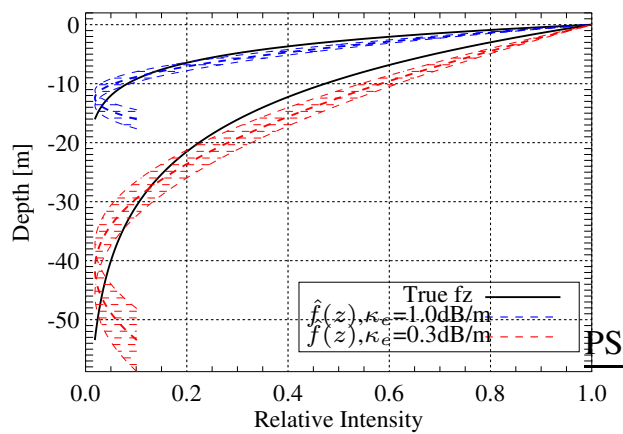

Fig. 1. Vertical profiles (solid) and their single-baseline Legendre series approximations (dashed) for a uniform volume with low $\kappa_{e}=0.3 \mathrm{~dB} / \mathrm{m}$ (in red, representative of L-band) and high $\kappa_{e}=1.0 \mathrm{~dB} / \mathrm{m}$ (in blue, X-band) extinctions. Variations in the derived profiles for errors in $d_{\text {pen }}$ of $\pm 10 \%$ are shown.

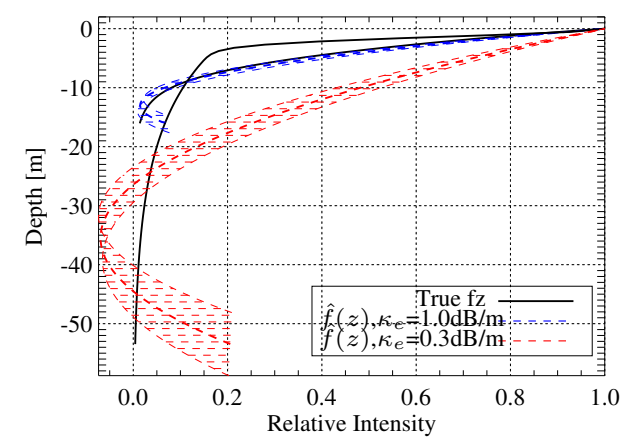

Fig. 2. Same as Fig. 1 for a volume-plus-ground scenario with $m=-3 \mathrm{~dB}$.

Figs. 1 and 2. It is seen that these penetration depth errors do not have a severe impact on the estimated profiles.

An error in the topographic phase $\phi_{0}$ will result in an uncompensated residual phase contribution to the normalized coherence $\gamma_{k}$ in Eq. 12. Because of poor conditioning of the diagonal $[F]$ matrix, any error or noise in $\gamma_{k}$ is strongly amplified [1]. Taking the standard deviation as a measure of the coherence error, the Cramér-Rao bound of the standard deviation of the coherence phase for $L$ effective looks is given by [7]:

$$
\sigma_{\phi}=\sqrt{\frac{1-\left|\gamma^{2}\right|}{2 L\left|\gamma^{2}\right|}} .
$$

To examine the influence of an error in $\phi_{0}$ on the estimated profile a similar geometry and radar parameters as in the experimental data (section IV) were used. For conciseness we present results only at a nominal $5 \mathrm{~m}$ baseline at L-band with $\theta=40^{\circ}, k_{z \mathrm{vol}}=0.08, \kappa_{e}=0.3 \mathrm{~dB} / \mathrm{m}\left(d_{\text {pen }}=-28 \mathrm{~m}\right),|\hat{\gamma}|=0.67$ and $L=38$. Assuming no error in the estimate of $d_{\text {pen }}$ and no noise in the coherence magnitude, the influence of an error in the topographic phase of $\pm 7.3^{\circ}$ (corresponding to the Cramér-Rao bound from Eq. 15) is given in Fig. 3, where the predicted $\sigma_{\phi}$ is close to the observed coherence phase standard deviation of $10^{\circ}$ in a 60x60 meter window surrounding the corner reflector.

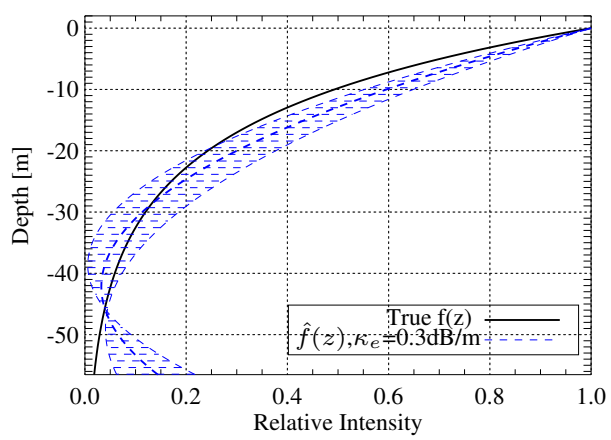

Fig. 3. Vertical profile (solid) and its single-baseline Legendre series approximation (dashed) for a uniform volume with extinction $\kappa_{e}=0.3 \mathrm{~dB} / \mathrm{m}$ in an L-band acquisition geometry. Variations in the derived profiles for errors in $\phi_{0}$ of $\pm 7.3^{\circ}$ (as determined by the Cramér-Rao lower bound) are shown.

The influence of fluctuations in coherence magnitude on the estimated Legendre coefficients was examined in [1], and the combined effect of errors in coherence magnitude and phase will be the subject of future work.

\section{EXPERIMENTAL DATA}

The test sites lie on the island of Nordaustlandet in northeastern Svalbard, Norway $\left(\sim 79-80^{\circ} \mathrm{N}, 20-27^{\circ} \mathrm{E}\right)$ in the subpolar glacial regime. Two sites were overflown, one in the accumulation zone near the summit of the Austfonna ice cap (referred to as 'Summit'), and one in the ablation zone near the Etonbreen outlet glacier ('Glacier').

The SAR data were obtained as part of the SVALEX (SVALbard airborne EXperiment) in April 2005, a joint project between the Microwaves and Radar Systems Institute of the German Aerospace Center (DLR) and the Alfred-Wegener Institute. Single-pass X-band data $(9.6 \mathrm{GHz})$ at VV polarization and repeat-pass fully-polarized L-band $(1.3 \mathrm{GHz})$ and P-band (350 MHz) data were collected using DLR's E-SAR system. Repeat-passes were flown at nominal baselines of 5, 10 and $15 \mathrm{~m}$ at an aircraft altitude of $5.5 \mathrm{~km}$. Incidence angles varied within the scene from $25-50^{\circ}$ and the data have a slant-range spacing of $1.5 \mathrm{~m}$ and an azimuth spacing of $1.32 \mathrm{~m}$ (X-band), $1.80 \mathrm{~m}$ (L-band) and $2.88 \mathrm{~m}$ (P-band) after multi-looking.

For calibration purposes and to determine the location of the ice surface, several corner reflectors and an approximately 200-m-long wire were deployed directly on the surface of the ice.

\section{RESULTS AND ANALYSIS WITH EXPERIMENTAL DATA}

The PCT method for a glacier geometry outlined in section II-B is applied to Pol-InSAR data at X-, L- and P-band to reconstruct vertical profiles of the upper firn layers on the subpolar Austfonna ice cap. The volume depth in Eqs. 5 and 7 was assumed to be $d_{\text {vol }}=C \cdot d_{\text {pen }}$ where $d_{\text {pen }}$ was inverted from Eq. 1 independently for each polarization after compensation of SNR and range spectral decorrelation. To obtain a complete 

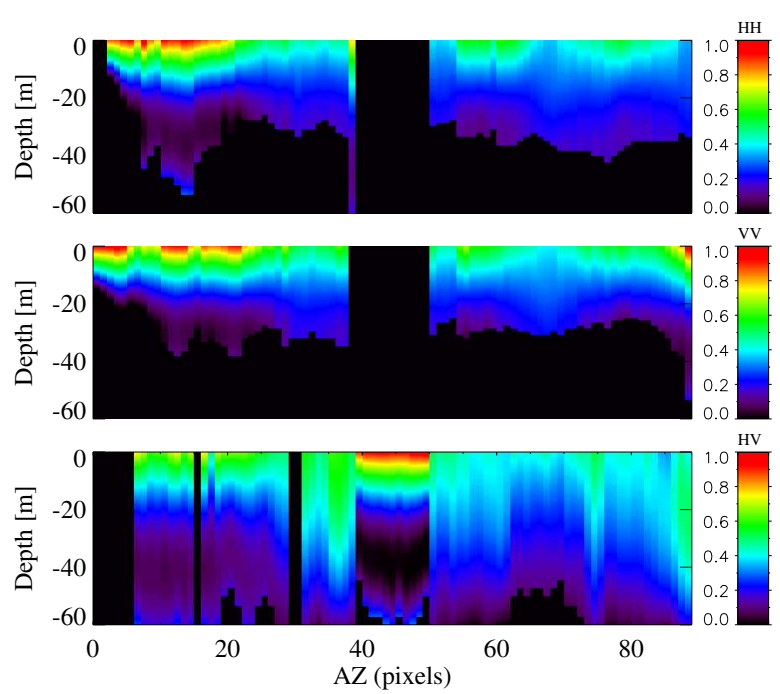

Fig. 4. Vertical structure profile cross-sections estimated using PCT for Glacier L-band data for a $5 \mathrm{~m}$ baseline.

profile $C$ should be varied with extinction and surface-tovolume scattering ratio, although for comparison of results between frequencies and polarizations we have set $C=2$ for all cases.

The results at L-band for a $5 \mathrm{~m}$ baseline $\left(k_{z \mathrm{vol}}=0.08\right)$ are shown in Fig. 4. The displayed cross-sections through azimuth intersect a corner reflector in the center of the image whose profile could not be inverted at $\mathrm{HH}$ and VV due to small $d_{\text {pen }}$ introducing instabilities in inversion of Eq. 12, and whose response at $\mathrm{HV}$ is stronger than that of the ice volume. At $\mathrm{HH}$ and VV the ice volume shows the same general trend of a strong response at the surface decreasing with depth until approximately -30 meters. At HV deeper estimates of penetration depth are the result of smaller coherence magnitudes $\left(\left|\hat{\gamma}_{\mathrm{HV}}\right|=0.48\right.$, whereas $\left|\hat{\gamma}_{\mathrm{VV}}\right|=0.67$ and $\left.\left|\hat{\gamma}_{\mathrm{HH}}\right|=0.64\right)$ which could be due to a lower extinction of $\sim 0.15 \mathrm{~dB} / \mathrm{m}$ and thus increased volume decorrelation at this polarisation, to additional uncompensated decorrelation effects, or to a combination of both. To avoid large variances, a coherence magnitude mask of 0.3 was applied and pixels with errors greater than $50 \%$ in the Legendre coefficients (where the fractional error is determined from [1]) were discarded and appear black in the profiles.

Further examples of vertical profiles at P-, L- and X-band are shown in Fig. 5. Note that these profiles are from different areas localized in two different test sites, but they reveal the overall trends present at each frequency. As in the crosssections from Fig. 4, there is a strong surface/near-surface response decreasing roughly exponentially with depth, with longer wavelengths having lower extinctions and thus deeper penetration depths. Because $f(z)$ may contain a mixture of surface and volume scattering which will in general depend on the polarization of the incident wave, additional differences in the profiles are seen between polarizations, where the marked
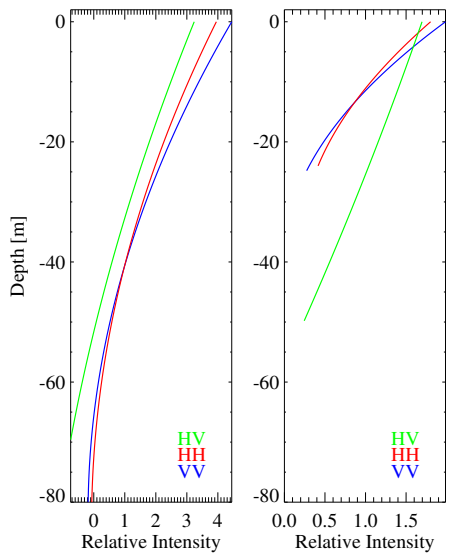

(a) P-band (b) L-band

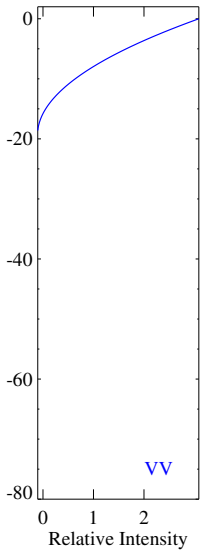

(c) X-band
Fig. 5. Vertical structure profiles estimated using coherence tomography for (a) Glacier P-band, B (baseline) $=5 \mathrm{~m}$, fully-polarized data (b) Glacier L-band, B=5 m, fully-polarized and (c) Summit X-band, B=1.7 m, VV.

difference between co- and cross-pols at L-band was discussed above. The shapes of these profiles are encouraging in that they display the exponential-type trend expected of a uniform volume and a uniform-volume-under-ground, justifying the use of these models for conventional Pol-InSAR analysis and parameter inversion for land-ice applications.

\section{SUMMARY}

In this paper polarization coherence tomography for vertical profile reconstruction was adopted for a glacier scenario. Estimation of the input parameters of volume depth and topographic phase was addressed for the land-ice case, and the sensitivity of the profiles to errors in the input parameters were simulated using realistic ground-to-volume scattering ratios and penetration depths. Examination of reconstructed vertical profiles revealed the same trend at all wavelengths and polarizations of a strong response at the surface exponentially decaying with increasing depth, confirming the exponential profile assumption of a uniform volume.

\section{REFERENCES}

[1] S. Cloude, "Polarization coherence tomography," Radio Sci., vol. 41, no. RS4017, 2006.

[2] S. R. Cloude and K. P. Papathanassiou, "Three-stage inversion process for polarimetric SAR interferometry," IEE Proc.-Radar Sonar Navig., vol. 150 , no. 3, pp. $125-134$, June 2003.

[3] S. Cloude, "Dual-baseline coherence tomography," IEEE Geosci. Remote Sens. Lett., vol. 4, no. 1, pp. 127 - 131, January 2007.

[4] W. Hoen, "A correlation-based approach to modeling interferometric radar observations of the Greenland ice sheet," Ph.D. dissertation, Department of Applied Physics, Stanford University, March 2001.

[5] J. J. Sharma, I. Hajnsek, and K. P. Papathanassiou, "Multi-frequency Pol-InSAR signatures of a subpolar glacier," in 3rd Intl. Workshop on Sci. and Appl. of SAR Polarimetry and Polarimetric Interferometry (PolInSAR2007), Frascati, Italy, 22-26 January 2007.

[6] F. T. Ulaby, R. K. Moore, and A. K. Fung, Microwave Remote Sensing, Active and Passive, Volume II: Radar Remote Sensing and Surface Scattering and Emission Theory. Reading, MA: Addison-Wesley, 1982.

[7] M. Seymour and I. Cumming, "Maximum likelihood estimation for SAR interferometry," in Proc. of the IEEE International Geosci. and Remote Sens. Symposium (IGARSS'94), 1994, pp. 2272-2275. 